S. SEGAWA

KODAI MATH. J.

17 (1994), 256-261

\title{
HARMONIC DIMENSION AND EXTREMAL LENGTH
}

\author{
Dedicated to Professor Nobuyuki Suita on his sixtieth birthday
}

\author{
By Shigeo Segawa
}

Consider an open Riemann surface $R$ with a single ideal boundary component. A subregion $V(\neq R)$ of $R$ is said to be an end of $R$ if $V$ is relatively noncompact in $R$ and the relative boundary $\partial V$ consists of finitely many analytic Jordan curves. Denote by $\mathscr{P}(V)$ the class of nonnegative harmonic functions on $V$ with vanishing boundary values on $\partial V$ :

$$
\mathcal{P}(V)=\{h \in H P(V): h \mid \partial V=0\},
$$

where $H P(V)$ is the class of nonnegative harmonic functions on $V$. The dimension of the linear space $\mathscr{Q}(V) \ominus \mathscr{P}(V)=\left\{h_{1}-h_{2}: h_{1}, h_{2} \in \mathscr{L}(V)\right\}$ is referred to as the harmonic dimension of $V$ (cf. Heins [4]), $\operatorname{dim} \mathscr{Q}(V)$ in notation. It is known that $\operatorname{dim} \mathscr{P}(V)$ does not depend on a choice of an end $V$ of $R$ (cf. [4]): $\operatorname{dim} \mathscr{Q}\left(V_{1}\right)$ $=\operatorname{dim} \mathscr{P}\left(V_{2}\right)$ for any pair $\left(V_{1}, V_{2}\right)$ of ends of $R$.

Denote by $O_{G}$ the class of open Riemann surfaces of null boundary and by $M$ the class of open Riemann surfaces $R \in O_{G}$ such that there exists an end $V$ of $R$ with $\operatorname{dim} \mathscr{Q}(V)=1$. In terms of Martin compactification an $R$ belongs to $M$ if and only if $R$ is of null boundary and the Martin boundary of $R$ consists of a single point (cf. e.g. Constantinescu and Cornea [3]). We are particularly interested in the following result by Heins [4] (see also [7]):

THEOREM A. Let $V$ be an end and $\left\{A_{n}\right\}$ be a sequence of mutually disjornt annuli in $V$ satisfying that $A_{n+1}$ separates $A_{n}$ from the ideal boundary of $V$ for every $n$. If the sum of moduli of $A_{n}$ diverges, then $\operatorname{dim} \mathscr{Q}(V)=1$.

We also denote by $O{ }_{S}^{\prime \prime}$ the class of open Riemann surfaces having a regular exhaustion $\left\{R_{n}\right\}_{n=0}^{\infty}$ such that each $A_{n}=R_{2 n}-\overline{R_{2 n-1}}(n=1,2, \cdots)$ is a doubly connected region and $\sum_{n=1}^{\infty} \bmod A_{n}=\infty$, where $\bmod A_{n}$ is the modulus of $A_{n}$. Then the above Heins' result is restated as

$$
O O_{S}^{\prime \prime} \subset M \text {. }
$$

* This work was partially supported by Grant-in Aid for Scientific Research, No. 04302006, Japanese Ministry of Education, Science and Culture.

Received June 5, 1993. 
The main purpose of this paper is to show that the inclusion $O_{S}^{\prime \prime} \subset M$ is struct.

In $\S 1$, we study harmonic dimension of an end which is a two-sheeted covering surface of the punctured unit disc $\{0<|z|<1\}$. In $\S 2$, applying a fact obtained in $\S 1$, we give an example which shows that the inclusion $O_{S}^{\prime \prime} \subset M$ is strict.

\section{Harmonic dimension of two-sheeted covering surfaces}

1.1. Consider two sequences $\left\{a_{n}\right\}$ and $\left\{b_{n}\right\}$ satisfying $0<b_{n+1}<a_{n}<b_{n}<1$ and $\lim _{n \rightarrow \infty} a_{n}=0$. Denote by $D=D\left(\left\{a_{n}\right\},\left\{b_{n}\right\}\right)$ the region $\Delta-\cup_{n=1}^{\infty} I_{n}$, where $I_{n}=\left[a_{n}, b_{n}\right]$ and $\Delta=\{0<|z|<1\}$. Take $N$ copies $D_{1}, D_{2}, \cdots, D_{N}$ of $D$. Joining the upper edge of $I_{n}$ in $D_{m}$ and the lower edge of $I_{n}$ in $D_{m+1}(\bmod . N)$ for every $n$, we obtain an $N$-sheeted covering surface $W=W\left(\left\{a_{n}\right\},\left\{b_{n}\right\}\right)$ of $\{0<|z|$ $<1\}$. We can view $W$ as an end of an $N$-sheeted covering surface of $\{0<|z|$ $\leqq \infty\}$. From Theorem A it follows that if $\sum_{n=1}^{\infty} \log \left(b_{n} / a_{n}\right)=\infty$ then $\operatorname{dim} \mathscr{Q}(W)$ $=1$. Heins also showed the following (cf. [4]):

THEOREM B. Let $D$ and $W$ be the same as above. Then (i) $\operatorname{dim} \mathscr{P}(W)$ is at most $N$ and (ii) $\operatorname{dim} \mathscr{P}(W)=N$ if the set $I=\cup_{n=1}^{\infty} I_{n}$ is sufficiently 'thin' at the point $z=0$ such as $\lim \sup _{R \ni x \rightarrow-0} \hat{R}_{\log (1 /|z|)}^{I}(x)<\infty$, where $\hat{R}_{\log (1 /|z|)}$ is the balayage of $\log (1 /|z|)$ on $\{|z|<1\}$ with respect to $I$.

Here and hereafter we restrict our attention to the case $N=2$. Thus $V$ is an end of a two-sheeted covering surface $R$ of $\{0<|z| \leqq \infty\}$ which is ramified over $\cup_{n=1}^{\infty}\left\{a_{n}, b_{n}\right\}$. Denote by $\pi$ the projection of $R$ onto $\{0<|z| \leqq \infty\}$. From (i) of Theorem B it follows that $\operatorname{dim} \mathscr{P}(W)=1$ or 2 , since $\mathscr{P}(W) \neq \emptyset$. We first prove the following which sharpens the above result in the case $N=2$ :

TheOREM 1. Suppose that $N=2$. Then $\operatorname{dim} \mathscr{Q}(W)=2$ if and only if the point $z=0$ is an irregular boundary point of the domain $D$ with respect to Dirichlet problem.

The proof is given in 1.2 and 1.3.

1.2. To begin with we state Heins' duality relation between harmonic dimensions and bounded harmonic functions. Heins [4] proved the following which is applied to the proof of Theorem 1:

THEOREM C. Let $V$ be an end. Then $\operatorname{dim} \mathscr{Q}(V)=1$ if and only if every bounded harmonic function on $V$ has a limit at the ideal boundary of $V$.

We are in the stage of proving 'if part' of Theorem 1. Denote by $u_{1}$ (resp. $u_{2}$ ) be the bounded harmonic function on $D_{1}$ (resp. $D_{2}$ ) with boundary values 1 
(resp. -1 ) on $\{|z|=1\}$ and 0 on $I=\bigcup_{n=1}^{\infty} I_{n}$. Let $w$ be a function on $W$ such that $w=u_{\imath}(i=1,2)$ on $D_{\imath}$. By the Schwarz reflection principle we see that $w$ can be considered as a bounded harmonic function on $W$. Since $u_{1}$ has a positive upper limit at $z=0$ by assumption (cf. e. g. Helms [5]), $w$ does not have a limit at the ideal boundary of $W$. Therefore Theorems $\mathrm{B}$ and $\mathrm{C}$ imply that $\operatorname{dim} \mathscr{P}(W)=2$.

1.3. Suppose that $z=0$ is a regular boundary point of $D$. We show that $\operatorname{dim} \mathscr{P}(W)=1$. We may assume that

$$
\sum_{n=1}^{\infty} \log \frac{a_{n}}{b_{n+1}}=\infty .
$$

In fact, otherwise, we have $\sum_{n=1}^{\infty} \log \left(a_{n} / b_{n}\right)=\infty$, which implies that $W$ satisfies the condition of Theorem A. Hence Theorem A yields that $\operatorname{dim} \mathscr{P}(W)=1$.

Let $p$ be a point in $W$. If $p$ belongs to the sheet $D_{\imath}(i=1,2)$, then we denote by $\bar{p}$ the point which belongs to $D_{\imath}$ and lies over $\overline{\pi(p)}$. Take an arbitrary $u \in H B(W)$, the space of bounded harmonic functions on $W$, and set $u^{*}(p)$ $=(1 / 2)(u(p)+u(\bar{p}))$. Observe that $u^{*}$ is a bounded harmonic function on $W$ satisfying $u^{*}(p)=u^{*}(\bar{p})$. We also set

$$
v(z)=\frac{1}{2}\left(u\left(p_{1}\right)+u\left(p_{2}\right)\right)
$$

for $z \in \Delta$, where $\pi^{-1}(z)=\left\{p_{1}, p_{2}\right\}$. Then $v$ is bounded and harmonic on $\Delta$, and hence on $\{|z|<1\}$. In particular we see that $v$ is continuous on $I \cup\{0\}$. Consider two functions $v_{i}=u^{*} \mid D_{\imath}(i=1,2)$. Note that $v(z)=u^{*}\left(p_{1}\right)=u^{*}\left(p_{2}\right)$ for every $z \in I$. Hence we have that $v_{i}$ can be viewed as a bounded harmonic function on $D$ with continuous boundary values $v \mid I \cup\{0\}$ on $I \cup\{0\}$. Therefore $v_{i}$ has a limit at $z=0$ by assumption.

We show that $u$ has a limit at the ideal boundary of $W$, which completes the proof by virtue of Theorem C. Put $J_{n}=\left[b_{n+1}, a_{n}\right](n=1,2, \cdots)$ and $J=$ $\bigcup_{n=1}^{\infty} J_{n}$. Consider two functions $u_{\imath}=u \mid D_{\imath}(i=1,2)$, which are viewed as bounded harmonic functions on $D$. Note that $u_{\imath}=v_{i}(i=1,2)$ on $J$. Hence, as proved in the preceding paragraph, we have

$$
\lim _{J \ni z \rightarrow 0}\left(u_{1}(z)-u_{2}(z)\right)=0 .
$$

For $r \in J$, denote by $\delta_{i}(r)$ the oscillation of $u_{\imath}$ on $\{|z|=r\}(i=1,2)$ :

$$
\delta_{i}(r)=\max _{|z|=r} u_{i}(z)-\min _{|z|=r} u_{i}(z) .
$$

We also denote by $\delta(r)$ the oscillation of $u$ on $\{|\pi(p)|=r\}$ :

$$
\delta(r)=\max _{|\pi(p)|=r} u(p)-\min _{|\pi(p)|=r} u(p) .
$$

It is easily seen that 


$$
\delta(r) \leqq \delta_{1}(r)+\delta_{2}(r)+\left|u_{1}(r)-u_{2}(r)\right| .
$$

Set $\delta_{n}=\min _{r \in J_{n}}\left(\delta_{1}(r)+\delta_{2}(r)\right)$. Then we have

$$
\delta_{n} \leqq \delta_{1}(r)+\delta_{2}(r) \leqq \sum_{j=1}^{2} \int_{0}^{2 \pi}\left|\frac{\partial u_{j}\left(r e^{i \theta}\right)}{\partial \theta}\right| d \theta
$$

for $r \in J_{n}$. Hence, by the Schwarz inequality and integration on $J_{n}$, we obtain

$$
\delta_{n}^{2} \mu_{n} \leqq 4 \pi \sum_{j=1}^{2} \int_{b_{n+1}}^{a_{n}} \int_{0}^{2 \pi} \frac{1}{r^{2}}\left|\frac{\partial u_{j}}{\partial \theta}\right|^{2} r d r d \theta \leqq 4 \pi \sum_{j=1}^{2} D_{n}\left(u_{\jmath}\right),
$$

where $\mu_{n}=\log \left(a_{n} / b_{n+1}\right)$ and $D_{n}\left(u_{\imath}\right)$ is Dirichlet integral of $u_{\imath}$ on $\left\{b_{n+1}<|z|<a_{n}\right\}$. Since $u$ has finite Dirichlet integral on $\left\{0<|\pi(p)|<a_{1}\right\}$, this implies that $\sum_{n=1}^{\infty} \delta_{n}^{2} \mu_{n}$ converges. Therefore it follows from (1) that $\liminf _{n \rightarrow \infty} \delta_{n}=0$. Hence, by (2) and (3), there exists a sequence $\left\{r_{n}\right\}$ such that $r_{n} \in J_{n}$ and $\lim _{n \rightarrow \infty} \delta\left(r_{n}\right)=0$. By means of maximum principle, this implies that $u$ has a limit at the ideal boundary. The proof is herewith complete.

\section{Extremal length of dividing curves}

2.1. Consider an open Riemann surface $R$ and its relatively compact subregion $F(\neq \emptyset)$. Let $\Gamma=\Gamma(R-\bar{F})$ be the family of closed curves in $R-\bar{F}$ which separate the ideal boundary of $R$ from $F$ and $\lambda(\Gamma)=\lambda(\Gamma(R-\bar{F}))$ be the extremal length of $\Gamma$. For the detail of extremal length, we refer to e.g. Ahlfors and Sario [2]. Denote by $O_{S}^{\prime}$ the class of open Riemann surfaces $R$ such that $\lambda(\Gamma(R-\bar{F}))=0$ for an $F$. It is well-known that the property $\lambda(\Gamma(R-\bar{F}))=0$ does not depend on a choice of $F$. Kusunoki [6] showed the following (see also Shiga $[8])$ :

THEOREM D. $O_{S}^{\prime \prime} \subset O_{S}^{\prime} \subset M$.

Set $a_{n}=e^{-n}\left(1-e^{-n^{2}}\right)$ and $b_{n}=e^{-n}$. For these sequences $\left\{a_{n}\right\}$ and $\left\{b_{n}\right\}$ and for $N=2$, let $D_{0}$ and $W_{0}$ be the region $D\left(\left\{a_{n}\right\},\left\{b_{n}\right\}\right)$ and the end $W\left(\left\{a_{n}\right\},\left\{b_{n}\right\}\right)$, respectively, which are considered in no. 1.1. We claim the following:

THEOREM 2. For the end $W_{0}$ given above, $\operatorname{dim} \mathscr{P}\left(W_{0}\right)=1$ and $\lambda(\Gamma)>0$, where $\Gamma$ is the family of closed curves in $W_{0}$ which separate the ideal boundary of $W_{0}$ from $\partial W_{0}$.

The proof is given in 2.2 and 2.3 .

2.2. Let $R$ be the two-sheeted covering surface of $\{0<|z| \leqq \infty\}$ which branches over $\cup_{n=1}^{\infty}\left\{a_{n}, b_{n}\right\}$ and $\varphi$ be the projection of $R$ onto $\{0<|z| \leqq \infty\}$. Theorem 2 implies that $R \in M-O_{S}^{\prime}$. Combining this with Theorem $\mathrm{D}$ it is immediately seen that $O_{S}^{\prime}<M$, and hence $O_{S}^{\prime \prime}<M$, where $<$ means strict inclusion. 
It is easily proved that $\operatorname{dim} \mathscr{Q}\left(W_{0}\right)=1$. In fact, since

$$
\sum_{n=1}^{\infty} \frac{n}{\log \left(4 /\left(b_{n}-a_{n}\right)\right)} \geqq \sum_{n=1}^{\infty} \frac{1}{n+3}=\infty,
$$

the Wiener criterion yields that the point $z=0$ is a regular boundary point of $D_{0}$ (cf. e.g. Tsuji [9]). Hence, by virtue of Theorem 1, we have the conclusion.

In order to prove that $\lambda(\Gamma)>0$, we provide the following lemma:

LEMma. Let $A_{n}$ be the annulus $\{|z|<1\}-\left(\left[0, a_{n}\right] \cup\left[b_{n}, 1\right]\right)$. Then $\bmod A_{n}$ $<\pi^{2} / n^{2}$.

In fact $A_{n}$ is conformally equivalent to the region $C-\left([-1,0] \cup\left[r_{n},+\infty\right]\right)$ where $r_{n}=\left(1-a_{n} b_{n}\right)\left(b_{n}-a_{n}\right) a_{n}^{-1}\left(1+b_{n}\right)^{-2}$. Hence we have $\bmod A_{n} \leqq \pi^{2} / \log \left(16 / r_{n}\right)$ (cf. e.g. Ahlfors [1]). It is easy to see that $\log \left(16 / r_{n}\right)>n^{2}$.

2.3. We start on the proof of $\lambda(\Gamma)>0$. Let $\Gamma_{n}$ be the family of $\gamma \in \Gamma$ satisfying that $\varphi(\gamma) \cap I_{n} \neq \emptyset$. Observe that

$$
\Gamma=\bigcup_{n=1}^{\infty} \Gamma_{n} .
$$

Set $\Gamma_{n}^{*}=\left\{\varphi(\gamma): \gamma \in \Gamma_{n}\right\}$. Then $\Gamma_{n}^{*}$ is a family of curves in $\Delta=\{0<|z|<1\}$. It is easily seen that

$$
2 \lambda\left(\Gamma_{n}\right) \geqq \lambda\left(\Gamma_{n}^{*}\right)
$$

for each $n$. Denote by $u$ the harmonic measure of the annulus $A_{n}$ with respect to the outer boundary and set $\rho=|\nabla u|$. We may assume that $\rho$ is defined on $\Delta$. Let $C_{n}$ be the family of closed curves in the annulus $A_{n}$ which separate the inner boundary from the outer boundary. Set $\bar{\gamma}=\{\bar{z}: z \in \gamma\}$ for $\gamma \in \Gamma_{n}^{*}$. Observe that $\gamma \cup \bar{\gamma}$ for each $\gamma \in \Gamma_{n}^{*}$ contains a closed curve which is approximated by a sequence in $C_{n}$. Hence we have

$$
4 \lambda\left(\Gamma_{n}^{*}\right) \geqq \inf _{\gamma \in \Gamma_{n}^{*}} \frac{(2 L(\gamma, \rho))^{2}}{A(\rho)} \geqq \inf _{\gamma \in \Gamma_{n}^{*}} \frac{L(\gamma \cup \bar{\gamma}, \rho)^{2}}{A(\rho)} \geqq \inf _{c \in C_{n}} \frac{L(c, \rho)^{2}}{A(\rho)}=\frac{2 \pi}{\bmod A_{n}},
$$

where $L(\gamma, \rho)=\int_{\gamma} \rho|d z|$ and $A(\rho)=\iint_{\Delta} \rho^{2} d x d y \quad(z=x+i y)$. By means of (4), (5) and Lemma, this yields that

$$
\lambda(\Gamma)^{-1} \leqq \sum_{n=1}^{\infty} \lambda\left(\Gamma_{n}\right)^{-1} \leqq \frac{4}{\pi} \sum_{n=1}^{\infty} \bmod A_{n} \leqq 4 \pi \sum_{n=1}^{\infty} \frac{1}{n^{2}}<\infty,
$$

which implies $\lambda(\Gamma)>0$. 


\section{REFERENCES}

[1] L. Ahlfors, Conformal Invariants, McGraw-Hill, 1973.

[2] L. Ahlfors and L. Sario, Riemann Surfaces, Princeton, 1960.

[3] C. Constantinescu And A. Cornea. Ideale Ränder Riemannscher Flächen, Springer, 1963.

[4] M. Heins, Riemann surfaces of infinite genus, Ann. of Math., 55 (1952), 296-317.

[5] L. Helms, Introduction to Potential Theory, Wiley-Interscience, 1969.

[6] Y. Kusunoki, On Riemann's period relations on open Riemann surfaces, Mem. Coll. Sci. Univ. Kyoto Ser. A. Math., 30 (1956), 1-22.

[7] S. Segawa, A duality relation for harmonic dimensions and its applications, Kodai Math. J., 4 (1981), 508-514.

[8] H. ShigA, On harmonic dimensions and bilinear relations on open Riemann surfaces, J. Math. Kyoto Univ., 21 (1981), 861-879.

[9] M. TsujI, Potential Theory in Modern Function Theory, Chelsea, 1975.

Department of Mathematics

Daido Institute of Technology

Daido, Minami, Nagoya 457

JAPAN 